\title{
Les exostosines: des protéines impliquées dans la biosynthèse des héparanes sulfates
}

Laurence Legeai-Mallet, Jacky Bonaventure

$>$ La maladie des exostoses multiples (HME), maladie héréditaire peu fréquente, est caractérisée cliniquement par la présence d'exostoses ou ostéochondromes se développant principalement au niveau des métaphyses des os longs. Elle affecte les enfants, de la petite enfance à la puberté, et à l'âge adulte les ostéochondromes peuvent dégénérer en chondrosarcomes $(0,5 \%$ à $5 \%$ des cas). Transmise sur le mode autosomique dominant, la maladie des exostoses multiples se caractérise par une hétérogénéité génétique puisque les études de liaison ont abouti à l'identification de 3 locus (EXT1, EXT2 et EXT3) localisés respectivement sur les chromosomes $8 q, 11 p$ et $19 p$. Des études moléculaires menées à la fois chez l'humain, la souris, le nématode et la drosophile ont permis d'identifier une nouvelle famille de gènes les EXT et EXTL (EXTlike). Des pertes d'hétérozygotie à un ou plusieurs locus EXT associées à la formation de chondrosarcomes confèrent à ces gènes $\varepsilon X T$ une fonction suppresseur
Inserm U. 393, Hôpital des Enfants Malades, 149, rue de Sèvres 75743 Paris Cedex 15, France.

de tumeur. Deux des gènes responsables de la maladie, EXT1 et EXT2 ont été clonés et séquencés $[1,2]$. Ils codent pour des protéines ubiquitaires de 746 ( $E X T 1$ ) et 718 ( $\varepsilon X T 2$ ) acides aminés appelées «exostosines» dans lesquelles ont été identifiés un domaine transmembranaire de type II, un petit domaine aminoterminal cytoplasmique et une grande région carboxy-terminale globulaire. Jusqu'en 1998, le rôle précis de ces protéines est resté méconnu. Plusieurs approches récentes ont conduit à des avancées significatives. Ainsi l'existence d'une lignée de cellules Sog9 déficientes en héparanes sulfates a permis de démontrer que le gène EXTI code pour une glycosyltransférase impliquée dans l'élongation des chaînes glycaniques des héparanes sulfates, une classe de protéoglycanes impliqués dans le contrôle de la croissance tumorale [3].

Des travaux complémentaires ont montré que la protéine codée par EXT2 est également une glycosyltransférase qui comme EXTl possède deux activités catalytiques, une activité glucuronyltransférase (GlcA-T) et une activité $\mathrm{N}$-acétylglucosaminyltransférase diphosphate (UDP). 
(GIcNAc-T) permettant la fixation alternée de résidus acide glucuronique et $\mathrm{N}$ acétyl glucosamine sur un tétrasaccharide de pontage lui même relié à la core protein par un résidu sérine. Parmi les EXTL, seule la protéine EXTL2 semble impliquée dans la biosynthèse des héparanes sulfates. Grâce à son activité $\alpha 1,4 \mathrm{~N}$ acétylglucosaminyltransférase elle transfère le premier résidu GIcNAc au tétrasaccharide de pontage de la core protein [4]. La protéine EXTL2 intervient donc en amont des protéines EXT 1 et EXT2 (Figure 1). Lorsque les protéines sont surexprimées séparément, elles sont localisées préférentiellement au niveau du réticulum endoplasmique. Pour assurer leur fonction polymérasique les deux protéines doivent former un complexe (hétéro-oligomère) qui se localise au niveau de l'appareil de Golgi, lieu de synthèse des héparanes sulfates [5]. Certains arguments supplémentaires suggèrent que les exostosines, pour être fonctionnelles, doivent appartenir à un complexe multienzymatique HS-polymérase comportant au moins une protéine chaperone et une autre glycosyltransférase. La position exacte des domaines catalytiques des deux exostosines n'a pas encore été déterminée de façon précise mais l'étude récente de cellules CHO (Chinese hamster ovarian) mutantes a permis de localiser l'activité GIcA-T au niveau de la partie centrale de la protéine codée par EXTI [6].

Des mutations ponctuelles non sens et faux sens affectant l'un ou l'autre des deux gènes $\varepsilon X T$ ont été détectées chez plus de $80 \%$ des patients atteints de maladie exostosante [7]. La plupart des mutations ayant pour conséquence la production de protéines tronquées, il en a été conclu qu'une haploinsuffisance d'un des gènes EXT était responsable de la formation des exostoses. Cependant, de façon inattendue, les souris invalidées pour le gène Extl sur un seul des deux allèles (Ext1+/-) n'ont développé aucune exostose alors que les embryons homozygotes pour l'invalidation ( $\left.\varepsilon x t^{-/-}\right)$mon- traient une létalité précoce à 6,5 jours de gestation [8]. Bien que les mutations de EXT1 et EXT2 chez l'humain semblent engendrer la formation d'exostoses histologiquement indiscernables, nous avons observé dans une étude récente portant sur 42 familles que les mutations de EXTl étaient associées aux formes les plus sévères de la maladie exostosante et que seules les exostoses résultant de mutations dans EXTI avaient la capacité de dégénérer en chondrosarcome [7]. Ces résultats démontrent une vraie corrélation entre la maladie et le gène muté et confirment les résultats biochimiques obtenus in vitro indiquant que les deux exostosines ne sont pas capables de se remplacer et que l'exostosine 1 semble jouer un rôle majeur dans la biosynthèse des héparanes sulfates. Parallèlement aux travaux in vitro sur la lignée Sog9, des études in vivo chez un mutant de drosophile appelé toutvelu ont apporté de nouveaux éléments quant au rôle des gènes EXT. Le gène tout-velu (ttv), homologue de EXT1, code pour une protéine à domaine transmembranaire de type II impliquée dans la biosynthèse des héparanes sulfates et semble contrôler la diffusion de la protéine Hedgehog (Hh) [9]. Chez les vertébrés, Indian Hedgehog (Ihh), un des trois membres de la famille Hh, est exprimé dans la plaque de croissance du cartilage dans les chondrocytes préhy-
1. Ahn J, Ludecke HJ, Lindow S, et al. Cloning of the putative tumour suppressor gene for hereditary multiple exostoses (EXTl). Nat Genet $1995 ; 11: 137-43$

2. Stickens D, Clines G, Burbee $D$, et al. The EXT2 multiple exostoses gene defines a family of putative tumour suppressor genes. Nat Genet $1996 ; 14: 25-32$.

3. McCormick G, Leduc Y, Martindale D, et al. The putative tumour suppressor EXTl alters the expression of cell-surface heparan sulfate. Nat Genet 1998 ; 19 : 158-61.

4. Kitagawa H, Shimakawa H, Sugahara K. The tumor suppressor EXT-like gene EXTL2 encodes an alphal,4-N-acetylhexosaminyltransferase that transfers $\mathrm{N}$-acetylgalactosamine and $\mathrm{N}$-acetylglucosamine to the common glycosaminoglycan-protein linkage region. The key enzyme for the chain initiation of heparan sulfate. J Biol Chem 1999 ; $274: 13933-7$.

5. Mc Cormick, Duncan G, Goutsos T, Tufaro F. The putative tumor suppressors EXTl and EXT 2 form a stable complex that accumulates in the Golgi apparatus and catalyzes the synthesis of heparan sulfate. Proc Natl Acad Sci USA 2000 ; 97 : 668 73.

6. Wei G, Bai X, Bame KJ, Koshy TI, Spear PG, Esko JD. Location of the glucuronosyltransferase domain in the heparan sulfate copolymerase EXTl by analysis of Chinese hamster ovary cells. J Biol Chem 2000 ; 275 : 27733 40.

7. Francannet $C$, Cohen-Tanugi A, LeMerrer M, Munnich A,
Bonaventure J, Legeai-Mallet L. Genotype-phenotype correlation in hereditary multiple exostoses. J Med Genet 2001 ; 38 : 430-4.

8. Lin X, Wei G, Shi Z, et al. Disruption of gastrulation and heparan sulfate biosynthesis in EXTl deficient mice. Dev Biol 2000 ; 224 : 299-

311.

9. Bellaiche $y$, The I, Perrimon N. Tout-velu is a Drosophila homologue of the putative tumour suppressor Extl and is needed for Hh diffusion. Nature 1998 ; 394 : 85-8.

10. Chung UI, Schipani $\varepsilon$, McMahon AP, Kronenberg HM. Indian hedgehog couples chondrogenesis to osteogenesis in endochondral bone development. J Clin Invest 2001 ; 107 : 295-304.

11. Ducy $P$, Contrôle génétique de la squelettogenèse. Med Sci 2001; 17 : 1242-51

12. Legeai-Mallet $L$, Rossi $A$, Benoist-Lasselin, et al. EXT 1 gene mutation induces chondrocyte cytoskeletal abnormalities and defective collagen expression in the exostoses. J Bone Mineral Res 2000 ; 15 : 1489-500.

13. Duncan G, McCormick C, Tufaro F. The link between heparan sulfate and hereditary bone disease : finding a function for the EXT family of putative suppressor tumor proteins. J Clin Invest 2001 ; 108 : 511-6. 
dernier jusqu'aux cellules cibles par un mécanisme qui implique les héparanes sulfates. Sachant que l'invalidation hétérozygote de $\varepsilon x t l$ affecte la fixation de Ihh au niveau de la surface cellulaire [8], I'hypothèse a été émise qu'un défaut de synthèse des héparanes sulfates présents à la surface des chondrocytes, suite à une mutation d'un des gènes $\varepsilon X T$, empêchait la prolifération des chondrocytes et provoquait une différenciation prématurée de ces cellules. Ce modèle est conforté par nos observations d'une expression ectopique de collagène de type $X$ (spécifique des chondrocytes hypertrophiques) [11] au niveau de certaines cellules de la zone proliférative suggérant que certains chondrocytes formant la partie cartilagineuse des exostoses se différencient de façon prématurée [12]. Cependant, la nature des cellules impliquées dans les stades les plus précoces de la formation des exostoses reste controversée. Alors que certains auteurs considèrent que les exostoses résultent d'une expansion clonale de chondrocytes porteurs d'une mutation somatique d'un des gènes EXT ou EXTL venant s'ajouter à la mutation germinale déjà présente [13], nous favorisons de notre coté l'hypothèse selon laquelle l'exostose dériverait de cellules mésenchymateuses qui prolifèreraient activement pour se différencier rapidement en chondrocytes hypertrophiques, puis en ostéoblastes, par un mécanisme de transdifférenciation. L'obtention de souris invalidées de façon tissu-spécifique pour l'un des gènes EXT devrait faciliter notre compréhension de la genèse des exostoses et de leur possible dégénérescence en tumeurs osseuses malignes. $\nabla$

Exostosines : tumor suppressor proteins involved in synthesis of heparan sulphate

\section{NOUVELLe}

\section{PML, un nouvel intermédiaire de l'effet antiviral de l'interféron}

Tarik Regad, Mounira K. Chelbi-Alix

$>$ Les interférons (IFN) sont des cytokines douées de nombreuses activités biologiques, telles que l'inhibition de la réplication virale, l'inhibition de la multiplication cellulaire, l'induction de l'apoptose ainsi que la modulation de la différenciation et de la réponse immunitaire. L'activité antivirale des IFN est à l'origine de leur découverte. Cette propriété a conduit à I'utilisation des IFN de type I1 dans le traitement de certaines infections virales, dues par exemple aux virus de papillomes et à ceux des hépatites $B$ et $C$.

L'IFN induit ses diverses activités biologiques en augmentant l'expression dans la cellule de plus de 200 protéines. Le rôle de la plupart de ces protéines n'est pas encore élucidé. L'expression de certaines est directement impliquée dans le mécanisme de l'action antivirale de I'IFN : c'est la protéine kinase dépendante de I'ARN double brin (PKR), le sys- tème 2'5'oligoadénylate synthétase /RNase $L$, et certaines formes de protéines Mx [1]. Cependant, l'invalidation des trois gènes $P K R$, RNase L et Mx ne suffit pas à abolir complètement la réponse antivirale de I'IFN $\alpha$, suggérant l'implication d'autres gènes dans la réponse antivirale.

Des données récentes montrent que PML (promyelocytic leukaemia), protéine induite par l'IFN, est responsable de certains effets antiviraux de cette cytokine.

\section{Structure et localisation de PML}

Le gène $P M L$ a été découvert dans la leucémie aiguë promyélocytaire associée à la translocation $t(15 ; 17)(\rightarrow)$ qui conduit à sa fusion avec le gène codant pour le récepteur de l'acide rétinoïque (RAR $\alpha$ ). PML code pour une protéine nucléaire qui est localisée soit de façon diffuse dans le nucléoplasme, soit sur les corps nucléaires (CN), structures de la matrice nucléaire dont la fonction est encore inconnue.

Plus d'une trentaine de protéines sont associées aux corps nucléaires, mais PML semble en être l'organisateur car ces structures sont absentes dans les cellules $P M L^{-/-}$[2]. En outre, PML est modifiée de façon covalente par SUMO-1 (small ubiquitin modifier) selon un processus qui ressemble à l'ubiquitinylation et pour lequel trois sites de liaison ont été identifiés $(\rightarrow)$. Cette $(\rightarrow) \mathrm{m} / \mathbf{s}$ modification par SUMO-1 2000, $\mathbf{n}^{\circ} \mathbf{1 1}$, est indispensable à l'intép. 1242 grité des corps nucléaires.

Ceux-ci sont aussi altérés dans certaines conditions physiopathologiques, en premier lieu dans la leucémie aiguë promyélocytaire, mais aussi en réponse au stress ou à certaines infections virales.

\section{$(\rightarrow) \mathrm{m} / \mathrm{s}$}

1994, n8-9, 1 Les IFN sont classés en deux types selon 817 leurs propriétés antigéniques et biologiques : les IFN de type I comprennent les
IFN $\alpha$ et IFNB et sont produits par toutes et 2001, IFN $\alpha$ et IFNß et sont produits par toutes $\begin{array}{ll}\mathbf{n}^{\circ} \mathbf{1}, \mathbf{p .} \mathbf{1 4} & \text { double-brin (db). L'IFN de type II ou IFN } \\ \text { est produit par les lymphocytes T et les }\end{array}$ cellules NK après stimulation par des antigènes ou des mitogènes. 